\title{
A microscopic model for D-wave pairing in the cuprates: what happens when electrons somersault?
}

\author{
Mona Berciu ${ }^{\mathrm{a}, *}$, Sajeev John ${ }^{\mathrm{b}}$ \\ ${ }^{a}$ Department of Electrical Engineering, Princeton University, Princeton, NJ 08544, USA \\ ${ }^{\mathrm{b}}$ Department of Physics, University of Toronto, 60 St. George Street, Toronto, Ontario, Canada M5S 1A7
}

\begin{abstract}
We present a microscopic model for a strongly repulsive electron gas on a $2 \mathrm{D}$ square lattice. We suggest that nearest-neighbor Coulomb repulsion stabilizes a state in which electrons undergo a "somersault" in their internal spin-space (spin-flux). When this spin- $\frac{1}{2}$ antiferromagnetic (AFM) insulator is doped, the charge carriers nucleate mobile, charged, bosonic vortex solitons accompanied by unoccupied states deep inside the Mott-Hubbard charge-transfer gap. This model provides a unified microscopic basis for (i) non-Fermi-liquid transport properties, (ii) mid-infrared optical absorption, (iii) destruction of AFM long-range order with doping, (iv) angled resolved spectroscopy (ARPES), and (v) $\mathrm{d}$-wave preformed charged carrier pairs. We use the configuration interaction (CI) method to study the quantum translational and rotational properties of such pairs. The CI method systematically describes fluctuation and quantum tunneling corrections to the Hartree-Fock approximation and recaptures essential features of the (Bethe ansatz) exact solution of the Hubbard model in 1D. For a single hole in the 2D AFM plane, we find a precursor to spin-charge separation. The CI ground state consists of a bound vortex-antivortex pair, one vortex carrying the charge and the other one carrying the spin of the doping hole. (C) 2001 Elsevier Science B.V. All rights reserved.
\end{abstract}

Keywords: Strongly correlated electron systems; High-temperature superconductivity; Topological magnetic excitations; Two-dimensional antiferromagnets

\section{Introduction}

In 1986 Bednorz and Muller [1] discovered that the perovskite $(\mathrm{BaLa})_{2} \mathrm{CuO}_{4}$ exhibits high-temperature superconductivity, with a critical temperature of up to $30 \mathrm{~K}$. Soon after, $\mathrm{La}_{2-x} \mathrm{Sr}_{x} \mathrm{CuO}_{4}$ and $\mathrm{YBa}_{2} \mathrm{Cu}_{3} \mathrm{O}_{7-x}$ were found to have superconducting critical temperatures of 35 and $95 \mathrm{~K}$, respective1y. Since then, many such compounds were found,

* Corresponding author. Fax: + 1-609-258-1840.

E-mail address: mberciu@princeton.edu (M. Berciu). including the $\mathrm{Tl}$ and $\mathrm{Hg}$ series. The current record $T_{\mathrm{c}}$ of $135 \mathrm{~K}$ ( $165 \mathrm{~K}$ under pressure) is found in the $\mathrm{HgBa}_{2} \mathrm{Ca}_{2} \mathrm{Cu}_{3} \mathrm{O}_{8}$ system. A typical phase diagram is shown in Fig. 1. The undoped parent is an insulator with long-range antiferromagnetic order. Extremely low doping $(x \approx 0.02$ charge carriers per site) leads to a complete destruction of the long-range AFM order, and a transition to an unusual non-Fermi-liquid metal. This unusual metal becomes superconducting, with the transition temperature $T_{\mathrm{c}}$ strongly dependent on the doping $x$. The maximum $T_{\mathrm{c}}$ is reached for dopings around $x=0.15$. For higher dopings the critical 


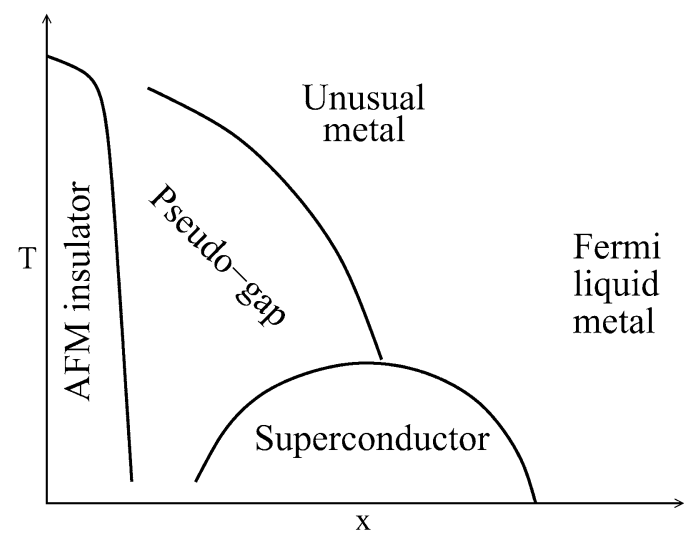

Fig. 1. Schematic phase diagram as a function of doping of high-temperature cuprate compounds.

temperature decreases to zero, and in the overdoped region a crossover towards a (non-superconducting) Fermi liquid takes place.

The effective two-dimensional Hamiltonian we use to describe the electrons residing in the $\mathrm{O}\left(2 \mathrm{p}_{\sigma}\right)$ $\mathrm{Cu}\left(3 \mathrm{~d}_{x^{2}-y^{2}}\right)$ orbitals of the isolated $\mathrm{CuO}_{2}$ plane is the generalized one-band Hubbard Hamiltonian

$$
\mathscr{H}=-\sum_{i, j, \sigma}\left(t_{i j} c_{i \sigma}^{\dagger} c_{j \sigma}+\text { h.c. }\right)+\sum_{i, j} V_{i j} n_{i} n_{j}
$$

where $c_{i \sigma}^{\dagger}$ creates an electron (in the orbital centered) at site $i$ with spin $\sigma, t_{i j}$ is the hopping amplitude from site $j$ to site $i$ on the square lattice, $n_{i} \equiv \sum_{\sigma} c_{i \sigma}^{\dagger} c_{i \sigma}$ is the total number of electrons at site $i$, and $V_{i j}$ is the Coulomb repulsion between electrons at sites $i$ and $j$. The dominant terms are the nearest-neighbor hopping $t_{i j}=t_{0}$ and the on-site Coulomb repulsion $V_{i i}=U / 2$. If only these two terms are considered, and we shift the chemical potential by $U$, this reduces to the widely studied Hubbard model.

In the undoped parent compound phase, there is one electron per orbital, and in the absence of interactions one would expect these compounds to be metallic, with a half-filled conduction band. Instead, from Fig. 1 we see that they are insulators with long-range AFM order. This is a strongly correlated electron system, with a large on-site repulsion term $(U>t)$, which causes electrons to become localized one per each orbital in order to avoid energetically expensive double occupancy. This obviously leads to insulating behavior, while the antiferromagnetism is simply a perturbational effect from the (small) hopping term [2]. However, as the plane is doped with holes, some of the previously filled orbitals are emptied, and electrons in nearby orbitals can freely hop into them, allowing for charge conduction. This is also seen from the phase diagram in Fig. 1, which shows that a very small amount of doping $x \approx 0.02$ completely destroys the LR AFM insulator phase, and the system becomes metallic (or superconductor, at lower temperatures). However, this metal has very unusual non-Fermi-liquid properties. Further doping leads to a crossover to a more conventional (but nonsuperconducting) Fermi-liquid metal in the extremely overdoped region.

Understanding the non-Fermi-liquid metal above the superconducting state is the central issue in the cuprate physics. Some of the most striking evidence of non-Fermi-liquid behavior is provided by angle resolved photo-emission spectroscopy (ARPES), which clearly shows the absence of quasiparticle peaks in the normal state. Equally compelling evidence is provided by resistivity measurements (For a review of transport properties see Ref. [3].) which reveal a scattering rate inversely proportional to the temperature $\tau \sim 1 / T$, extending over a range of up to $700 \mathrm{~K}$ [4-6]. In an ordinary Fermi liquid, electron-electron scattering gives a $T^{2}$ dependence of the relaxation rate, related to the quadratic energy dependence of the quasiparticle lifetime $1 / \tau \sim\left|\varepsilon-\varepsilon_{\mathrm{F}}\right|^{2}$. This is a hallmark of a Fermi liquid [7]. In fact, the canonical $T^{2}$ behavior is indeed observed in the extremely overdoped region, already identified as a Fermi liquid (For a review of optical properties see [8]). But its absence in the intermediate doping region of the unusual metal, combined with the absence of quasiparticle peaks in the ARPES data clearly show that this unusual metal is not a Fermi liquid. Then, a natural question arises. If the charge carriers of the unusual metal are not the quasiparticlelike charge carriers of a Fermi liquid, what is their nature?

Some clues are provided by experiments. Hall measurements in the underdoped regime tell us 
that the charge carriers are positively charged and their density equals the doping, i.e. the hole density (For a review of the Hall effect see [9].) (Deviations of both the sign and the density scaling are observed for some compounds in the overdoped regions). Optical measurements reveal the appearance, with doping, of a low-frequency Drude tail, which suggests the existence of free, or very mobile charge carriers [8]. These optical measurements confirm both the anomalous $1 / T$ scattering rate of these charge carriers, as well as the fact that their density equals the doping. Together, these measurements suggest that each hole introduced in the $\mathrm{CuO}_{2}$ layer evolves into a mobile, positively charged, carrier (but not a quasiparticle). However, both types of measurements show other anomalous behavior as well. The Hall coefficient has a strong, $1 / T$ temperature dependence, which is very puzzling, since neither the density nor the charge of the carriers is temperature dependent, and neither is the Lorentz force. The optical measurements reveal that the Drude tail is accompanied by the appearance of a broad, mid-infrared band deep inside the Mott-Hubbard gap [8]. This mid-infrared band develops with doping, and signifies the existence of localized levels inside the Mott-Hubbard gap. Unlike the Drude tail which collapses below $T_{\mathrm{c}}$ to a $\delta(\omega)$ function, this mid-infrared response persists unchanged both above and below $T_{\mathrm{c}}$. Magnetic measurements indicate that although the LR AFM is completely suppressed above $x \approx 0.02$, strong short-range AFM correlations persist, with a correlation length roughly equal to the average distance between the holes (charge carriers) (For a review of magnetic properties see [10]). Neutron scattering indicates the appearance of incommensurate peaks in the magnetic structure factor, with a shift from the $\operatorname{AFM}(\pi / a, \pi / a)$ vector varying linearly with doping for $0.02<x<0.12$ and then saturating. The four incommensurate peaks are arranged diagonally for $x<0.05$ and then rotate by $45^{\circ}$ for $x>0.05$ [11]. The doping dependence of all these features clearly indicates that magnetism is crucial to the entire phenomenology.

More puzzling behavior appears in the superconducting state. Flux quantization clearly proves that pairing does take place, and the unit of charge in the superconducting condensate is 2e. ARPES and phase-sensitive measurements have shown that the superconducting gap has d-wave symmetry, and this is believed to mirror the symmetry of the "Cooper-pair" wave function to internal rotations. Penetration depth measurements show that the density of superfluid in the limit $T \rightarrow 0$ scales with doping. This means that the "Cooper-pairs" must, in fact, be formed from the positive charge carriers of the unusual metal, not from electrons, as in conventional BCS theory (the density of electrons is $1-x$, not $x$ ). This underscores the need to identify the charge carrier of the unusual metal, but leads to another question, namely, how does strong pairing (leading to high superconducting temperatures) occur in a system dominated by strong Coulomb repulsion?

In this paper we describe a microscopic model which offers simple and compelling answers to the above questions, as well as to other puzzling features described above. Unlike other approaches which assume that the fundamental quantum degrees of freedom of the many-electron system are conventional and that the resulting phenomenology is an "emergent law of nature" arising from the complexity of the system, we propose that there is a hidden fundamental law of Nature. This fundamental law of nature expresses a novel dynamical degree of freedom, namely that an electron can perform a "somersault" in its internal space of Euler angles (when considered as a rigid body of non-zero volume) as the electron traverses a closed loop in external coordinate space. The result is a new quantum number for the many-electron system (the eigenvalues of the spin-flux), which we propose is as fundamental as the existence of the spin $-\frac{1}{2}$ itself. We argue that the electronic somersault (spin-flux) is dynamically generated through electromagnetic interactions and in particular, the off-diagonal part of the Coulomb repulsion between electrons. In Section 2 we introduce the spin-flux Hamiltonian, which we propose as the appropriate Hamiltonian describing the isolated $\mathrm{CuO}_{2}$ plane. In Section 3 we apply Hartree-Fock approximation (HFA) and the configuration interaction (CI) method to the spin-flux Hamiltonian and we identify both the mobile bosonic charge carrier as well as the nature and symmetry of the strong pairing interaction between such charge 
carriers. We provide a physical justification for the accuracy of the CI approximation and we explicitly demonstrate this by comparison with the exact solution of the 1D Hubbard model [12]. Finally, in Section 4 we discuss the comparison of our model and its results with the experimental findings and draw some final conclusions.

\section{The spin-flux model}

The neglect of the dynamical consequences of longer range Coulomb interaction $\left(V_{i j}=0\right.$, if $\left.i \neq j\right)$, in the generalized Hubbard model of Eq. (1), is based on the assumption of uniform charge distribution and on the Fermi-liquid theory notion of screening of the effective electron-electron interaction. However, Fermi-liquid theory fails to explain many of the crucial features of the high- $T_{\mathrm{c}}$ cuprates. In our description, we include the nearestneighbor Coulomb repulsion, which we assume is on the energy scale of $t$. This has important dynamical consequences in our model and cannot simply be absorbed into the Madelung constant. In particular, it leads to the generation of spin-flux, an entirely new type of broken symmetry in the many-electron system, which we show leads naturally to bosonic charge carriers in the form of meron-vortices, non-Fermi-liquid behavior and a strong attractive pairing force between holes in the AFM background.

The concept of spin-flux is closely related to the existence of the spin $-\frac{1}{2}$ particles in nature. The spin of a physical electron may be regarded as arising from the quantization of a classical, symmetric spinning top [13] whose kinematical properties are described by a set of three independent Euler angles. These Euler angles constitute a continuous manifold, the group manifold of $\mathrm{SO}(3)$. Unlike the manifold $\mathrm{S}_{2}$ (the surface of a unit sphere) describing the orientation of a classical magnetic moment, the group manifold of $\mathrm{SO}(3)$ is not topologically simply connected. According to the axioms of quantum mechanics any physical wave function must be everywhere continuous and differentiable. For this to be satisfied on a simply connected manifold, the wave function must be single valued. Consequently the $\mathrm{O}(3)$ nonlinear sigma model describes integer
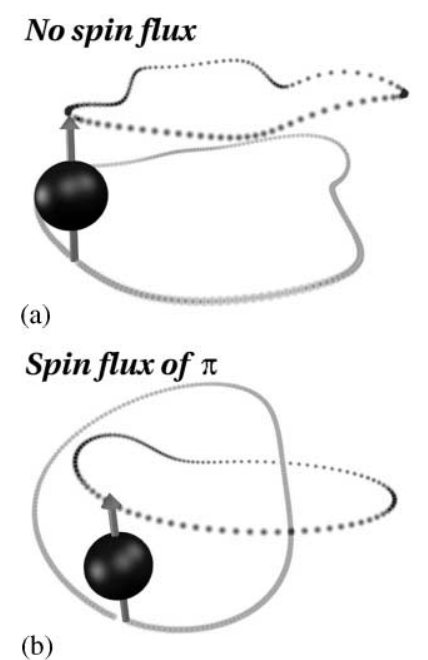

Fig. 2. Conventional (a) vs. spin-flux (b) trajectory. In the latter case, the spin rotates by $2 \pi$ (somersault) as it encircles the path in real space.

spins. The doubly connected group manifold of $\mathrm{SO}(3)$, however, can accommodate two-valued wave functions which are everywhere continuous and differentiable. This leads to half-integer spins. In order to accommodate spin $-\frac{1}{2}$, the $\mathrm{O}(3)$ nonlinear sigma model must be supplemented with a magnetic monopole which is placed at the center of the sphere $S_{2}[14,15]$. The charge of this monopole corresponds to a quantum of the third Euler angle in the parameterization of the $\mathrm{SO}(3)$ group manifold.

Spin-flux arises when the electron executes a topologically nontrivial path within its internal space of Euler angles while it traverses a closed loop in the external coordinate space. The internal path is one that cannot continuously be deformed to zero and corresponds to a $2 \pi$ rotation (somersault) in the space of Euler angles. Another depiction of this process (see Fig. 2) is seen by considering distinct points within the spinning top and following their trajectories as the electron executes a closed path in external coordinate space. In a non-spin-flux circuit, the two trajectories are unlinked, whereas in a spin-flux circuit (involving a somersault) the two trajectories are linked. We propose that this novel possibility represents a hidden but fundamental Law of Nature which has 
not been considered in conventional treatments of many-body theory. Spin-flux corresponds to a fundamentally new quantum number in a manyelectron system and requires the extension of the conventional many-electron Hilbert space. We suggest that this simple addition (at a fundamental level) to the dynamical degrees of freedom of interacting electrons an a two-dimensional lattice leads to a unified, microscopic explanation of a large variety of experiments on the cuprates.

In order to describe the above physics from our starting Hamiltonian

$$
\begin{aligned}
\mathscr{H}= & -t_{0} \sum_{\langle i, j\rangle \sigma}\left(c_{i \sigma}^{\dagger} c_{j \sigma}+\text { h.c. }\right)+U \sum_{i} n_{i \uparrow} n_{i \downarrow} \\
& +V \sum_{\langle i, j\rangle} n_{i} n_{j}
\end{aligned}
$$

we introduce bilinear combination of electron operators $\Lambda_{i j}^{\mu} \equiv c_{i \alpha}^{\dagger} \sigma_{\alpha \beta}^{\mu} c_{j \beta}, \mu=0,1,2,3$, for $i \neq j$ (summation over multiple indexes is assumed). Here $\sigma^{0}$ is the $2 \times 2$ identity matrix and $\boldsymbol{\sigma} \equiv\left(\sigma^{1}, \sigma^{2}, \sigma^{3}\right)$ are the usual Pauli spin matrices. The notation $\langle i, j\rangle$ means that the sites $i$ and $j$ are nearest neighbors. The quantum expectation value $\left\langle>\right.$ of the $\Lambda_{i j}^{\mu}$ operators are associated with charge-currents $(\mu=0)$ and spin-currents $(\mu=1,2,3)$. Non-vanishing charge currents lead to appearance of electromagnetic fields, which break the time-reversal symmetry of the Hamiltonian. Experimentally, this does not occur in the cuprates. In the following, we adopt the ansatz that there is no charge current in the ground state $\Lambda_{i j}^{0}=0$ but circulating spin-currents may arise and take the form $\Lambda_{i j}^{a}=2 t_{0} / V \mathrm{i} \Delta_{i j} \hat{n}_{a}, a=1,2,3$, where $\left|\Delta_{i j}\right|=\Delta$ for all $i$ and $j$, and $\hat{n}$ is a unit vector. These spin-currents provide a transition state to the uniform spin-flux mean field that we use in this paper. In principle, non-uniform states of spin-flux may arise, in which $\left|\Delta_{i j}\right|$ has a nontrivial dependence on $i$ and $j$. One such case was discussed earlier [14,15], in which skyrmion textures in the AFM background carry quantized units of spin-flux. In this case $\Delta_{i j}$ is a dynamical variable. However, for the purpose of this paper, we consider only a uniform, static, mean-field configuration of the spin-flux.

Using the Pauli spin-matrix identity, $\frac{1}{2} \sigma_{\alpha \beta}^{\mu}\left(\sigma_{\alpha^{\prime} \beta^{\prime}}^{\mu}\right)^{*}$ $=\delta_{\alpha \alpha^{\prime}} \delta_{\beta \beta^{\prime}}$, it is possible to rewrite the nearest- neighbor electron-electron interaction terms as $n_{i} n_{j}=2 n_{i}-\frac{1}{2} \Lambda_{i j}^{\mu}\left(\Lambda_{i j}^{\mu}\right)^{+}$. We neglect fluctuations in the spin-currents, and use the mean-field factorization to replace $\Lambda_{i j}^{\mu}\left(\Lambda_{i j}^{\mu}\right)^{+} \rightarrow\left\langle\Lambda_{i j}^{\mu}\right\rangle\left(\Lambda_{i j}^{\mu}\right)^{+}+$ $\Lambda_{i j}^{\mu}\left\langle\Lambda_{i j}^{\mu}\right\rangle^{*}-\left\langle\Lambda_{i j}^{\mu}\right\rangle\left\langle\Lambda_{i j}^{\mu}\right\rangle^{*}$. Thus, the quartic nearest-neighbor Coulomb interaction term is reduced to a quadratic term that is added to the hopping term leading to the effective Hamiltonian

$$
\mathscr{H}=-t \sum_{\substack{\langle i, j\rangle \\ \alpha \beta}}\left(c_{i \alpha}^{\dagger} T_{\alpha \beta}^{i j} c_{j \beta}+\text { h.c. }\right)+U \sum_{i} n_{i \uparrow} n_{i \downarrow} .
$$

Here, $T_{\alpha \beta}^{i j} \equiv\left(\delta_{\alpha \beta}+\mathrm{i} \Delta_{i j} \hat{n} \cdot \sigma_{\alpha \beta}\right) / \sqrt{1+\Delta^{2}}$ are spindependent $\mathrm{SU}(2)$ hopping matrix elements defined by the mean-field theory, and $t=t_{0} \sqrt{1+\Delta^{2}}$. In deriving Eq. (3), we have dropped constant terms which simply change the zero of energy as well as terms proportional to $\sum_{i} n_{i}$ which simply change the chemical potential. It was shown previously [14-16] that the ground-state energy of the Hamiltonian of Eq. (3) depends on the SU(2) matrices $T^{i j}$ only through the plaquette matrix product $T^{12} T^{23} T^{34} T^{41} \equiv \exp (\mathrm{i} \hat{n} \cdot \sigma \Phi)$. Here, $\Phi$ is the spinflux which passes through each plaquette and $2 \Phi$ is the angle through which the internal coordinate system of the electron rotates as it encircles the plaquette. Since the electron spinor wave function is two-valued, there are only two possible choices for $\Phi$. If $\Phi=0$ we can set $T_{\alpha \beta}^{i j}=\delta_{\alpha \beta}$ and the Hamiltonian (3) describes conventional ordered magnetic states of the Hubbard model. The other possibility is that a spin-flux $\Phi=\pi$ penetrates each plaquette, leading to $T^{12} T^{23} T^{34} T^{41}=-1$. This means that the one-electron wave functions are antisymmetric around each of the plaquettes, i.e. that as an electron encircles a plaquette, its wave function in the internal spin space of Euler angles rotates by $2 \pi$ in response to strong interactions with the other electrons. In effect, the electron performs an internal "somersault" as it traverses a closed path in the $\mathrm{CuO}_{2}$ plane $[14,15]$. This spin-flux phase is accompanied by a AFM local moment background (with reduced magnitude relative to the AFM phase of the conventional Hubbard model). In the spin-flux phase, the kinetic energy term in Eq. (3) exhibits broken symmetry as though a spin-orbit interaction has been added. In the presence of charge carriers this mean field is unstable to the proliferation of 
topological fluctuations (magnetic solitons) which eventually destroy AFM long-range order. In this sense, the analysis which we present below goes beyond simple mean-field theory. The quantum dynamics of these magnetic solitons described by the configuration interaction (CI) method, corresponds to tunneling effects not contained in the Hartree-Fock approximation. For simplicity, throughout this paper we assume that the meanfield spin-flux parameters $T^{i j}$ are given by the simplest possible choice $T^{12}=-1, T^{23}=T^{34}=$ $T^{41}=1$ (for more details, see Ref. [17]). In order to go beyond a mean-field description of the spin-flux, these matrices may also be treated as dynamical variables. In this paper, we go beyond mean-field theory in describing the antiferromagnetic degrees of freedom but restrict ourselves to a mean-field model of the spin-flux.

The mean-field ground state of the undoped spin-flux model is an AFM Mott insulator. It is

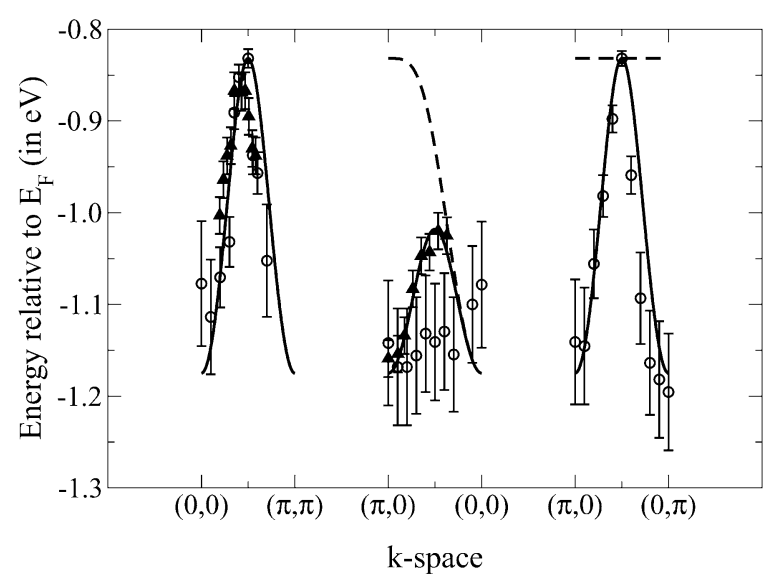

Fig. 3. A comparison between the experimentally determined $E(\boldsymbol{k})$ quasiparticle dispersion relation, from angle resolved photoemission studies (ARPES), for the insulating $\mathrm{Sr}_{2} \mathrm{CuO}_{2} \mathrm{Cl}_{2}$ and the HF AFM spin-flux model dispersion relation (full line) and the HF AFM conventional Hubbard model dispersion relation (dashed line). Three directions in $\boldsymbol{k}$-space are shown: $(0,0)$ to $(\pi, \pi),(\pi, 0)$ to $(0,0)$ and $(\pi, 0)$ to $(0, \pi)$. While the peak on the $(0,0)$ to $(\pi, \pi)$ is equally well described in both models, the mean-field spin-flux model gives a much better agreement for the $(\pi, 0)$ to $(0,0)$ and $(\pi, 0)$ to $(0, \pi)$ directions. The fitting corresponds to $U=2.01 \mathrm{eV}, t=0.29 \mathrm{eV}$ for the spin-flux phase, and $U=1.98 \mathrm{eV}, t=0.21 \mathrm{eV}$ in the conventional phase. The experimental results are the ARPES results of Ref. [18] (circles) and Ref. [19] (triangles). interesting to note that the quasiparticle dispersion relation obtained in the presence of the spin-flux [17] accurately recaptures the dispersion as measured through angle-resolved photo-emission spectroscopy (ARPES) in a compound such as $\mathrm{Sr}_{2} \mathrm{CuO}_{2} \mathrm{Cl}_{2}$ [18] (see Fig. 3). There is a large peak centered at $(\pi / 2, \pi / 2)$ with an isotropic dispersion relation around it, observed on both the $(0,0)$ to $(\pi, \pi)$ and $(0, \pi)$ to $(\pi, 0)$ lines. The spin-flux model in HFA exhibits another smaller peak at $(0, \pi / 2)$ which has been observed in more recent experimental data $[19,20]$. The quasiparticle dispersion relation of the conventional Hubbard model $\left(T^{12}=T^{23}=T^{34}=T^{41}=1\right)$ has a large peak at $(\pi / 2, \pi / 2)$ on the $(0,0)$ to $(\pi, \pi)$ line (see Fig. 3$)$, but it is perfectly flat on the $(0, \pi)$ to $(\pi, 0)$ line (which is part of the large nested Fermi surface of the conventional 2D Hubbard model). Also, it has a large crossing from the upper to the lower band-edge on the $(0,0)$ to $(0, \pi)$ line. Both this dispersion relation and the very similar one of the $t-J$ model (see Ref. [18]) are in contradiction to ARPES data.

\section{Doping induced meron-vortex solitons}

\subsection{The static Hartree-Fock approximation}

The HF results for the undoped AFM ground state of the spin-flux Hamiltonian are in good agreement with experimentally measured dispersion (see Fig. 3). The azimuthal symmetry of the dispersion relations about the Fermi points plays a key role in determining the symmetries of doping-induced magnetic configurations. This is more straightforward to see in a simpler, continuum version of the model, obtained by letting the lattice constant $a \rightarrow 0$ (see Refs. [21,22]). Since the dispersion relation near the Fermi point $\boldsymbol{q}=(\pi / 2 a, \pi / 2 a)$ is isotropic, the dependence on $\boldsymbol{k}=\boldsymbol{K}-\boldsymbol{q} \rightarrow$ $-\mathrm{i} \nabla_{r}$ of the continuum HF equations is such that it preserves rotational invariance. As a result, the 2D HF equation reduces trivially to a $1 \mathrm{D}$ radial equation, with a structure very similar to that of the 1D differential HF equation obtained for the 1D Hubbard model [21,22]. Once this radial 1D solution is found, the $2 \mathrm{D}$ configuration is simply generated through a $2 \pi$ rotation about an axis 


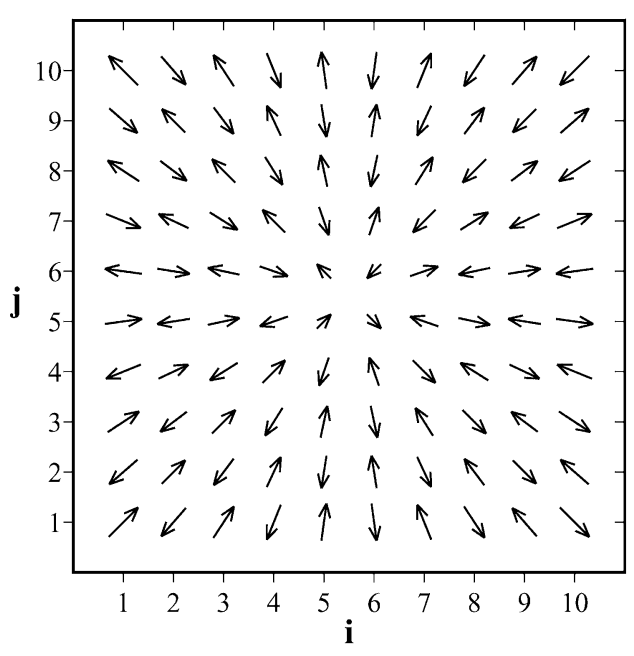

Fig. 4. Self-consistent spin distributions of a $10 \times 10$ lattice with a meron-vortex in the spin-flux phase. The core of the meron is localized in the center of a plaquette, in the spin-flux phase (in the conventional phase, the core of the meron-vortex is localized at a site). This excitation has a topological winding number 1 , since the spins on either sublattice rotate by $2 \pi$ on any curve surrounding the core. The magnitude of the staggered magnetic moments is slightly diminished near the vortex core but is equal to that of the undoped AFM background far from the core. The hole is localized in the vortex core.

perpendicular to the $2 \mathrm{D}$ plane. As a result, there is a close analogy between solutions obtained for the 1D Hubbard model and for the 2D spin-flux model, in all our investigations [12,21-23].

In the corresponding discrete model, the relevant doped configuration is the meron-vortex (see Fig. 4). The doping hole is trapped in the core of a magnetic vortex, which indeed has azimuthal symmetry. The bound level on which the hole is trapped can be shown $[17,22]$ to be split from the top of the valence band and drawn deep inside the Mott-Hubbard gap. As a result, the meron-vortex is a charged boson. This can be inferred by direct inspection of Fig. 4, which shows a configuration with total spin zero and a positive charge trapped in its core. An argument based on the electronic structure, identical to the one offered for the charged bosonic domain walls of the polyacetylene, also holds $[17,21,22,24]$. The parallel to the quasione-dimensional $1 \mathrm{D}$ polyacetylene is again a reflection of azimuthal symmetry which reduces the 2D continuum model to a $1 \mathrm{D}$ radial equation. Clearly,

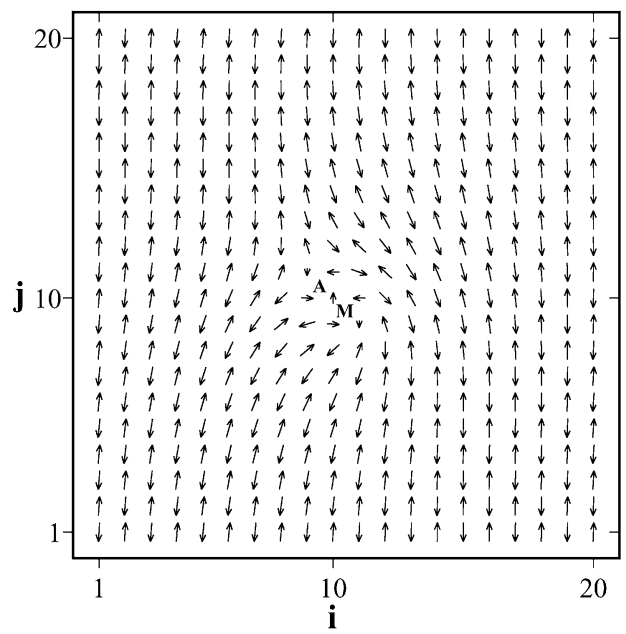

Fig. 5. Self-consistent spin distribution for a tightly bound meron-antimeron pair. The meron (M) and the antimeron (A) are localized on neighboring sites. The total winding number of the pair is zero. The magnetic AFM order is disturbed only in the region where the vortices are localized. The attraction between holes is of topological nature and on long length scale is stronger than unscreened Coulomb repulsion between charges. The doping charge is mostly localized on the two plaquettes containing the meron and antimeron cores. The two holes localized in the vortex cores are responsible for the fact that the meron-antimeron pair does not collapse.

the isotropic dispersion about the Fermi points is responsible for the appearance of bosonic charge carriers. They are very unlike quasiparticle charge carriers, which carry both spin and charge together. Thus, one would expect a metal with such bosonic charge carriers to have intrinsically different properties from those of a Fermi-liquid metal.

A look at the spin-configuration in Fig. 4 also shows that this cannot be realized if cyclic boundary conditions (CBC) are imposed. With them, the self-consistent solution found in the presence of one hole is a very different configuration called the spin-bag, which is a rather immobile quasiparticlelike configuration (carrying both charge $+e$ and spin- $\frac{1}{2}$ ) $[17,23]$. However, for more than one hole added to the AFM plane, the HF ground state of the spin-flux Hamiltonian always shows meronvortices created through doping, even with $\mathrm{CBC}$ [17]. The simple reason for this is that while a single isolated meron-vortex is incompatible with CBC, meron-antimeron pairs are not (see Fig. 5 for 
a typical meron-antimeron configuration). In fact, the nucleation of merons and antimerons in pairs also solves another problem, related to the energetic cost of creating a meron. It is straightforward to prove that the energy of a single-meron configuration increases logarithmically with its size [17]. Beyond a certain (large) separation it is energetically favorable for the meron-antimeron pair to collapse into a pair of charged spin bags. In the conventional Hubbard model (with no spin-flux) spin bags are favored at all separations. In the spin-flux phase, the farther the meron is from the antimeron, the more spins in between are rotated by the vortices, and the excitation energy increases. As a result, an isolated pair tends to be as closely bound as possible. If the vortices were uncharged, at low temperatures they would annihilate each other. However, the holes localized in the vortex cores lead to a very strong short-range Coulomb repulsion which prevents the pair annihilation, thus making the pair stable. It is worth noting that even in the complete absence of screening, at long distances the $1 / r$ Coulomb repulsion would be overcome by the $\ln (r)$ attraction between vortices, leading to a stable bound pair.

To conclude, we see that even at the static HF level, charge carriers in the spin-flux phase exhibit bosonic nature, and a strong pairing attraction to other charge carriers. This attraction is of magnetic origin, arising from exchange energy lost by spins which are no longer perfectly AFM aligned. The importance of the bosonic meron-vortex excitations becomes even more apparent when we consider fluctuation and tunneling corrections to the HF approximation. These correspond to translational motion of the charged vortices. It turns out that charged meron-vortices have an effective mass comparable to that of the band electron. As seen from the CI method, they are much more mobile than the very heavy spin-bags.

\subsection{Fluctuations and tunneling: the configuration interaction method}

We now discuss how to improve the mean-field description above. Given a complete basis of states $\left\{\left|\phi_{i}\right\rangle\right\}$ spanning the $N$-body Hilbert space, the exact $N$-body ground-state wave function can be written as $|\Psi\rangle=\sum_{i} \alpha_{i}\left|\phi_{i}\right\rangle$. The coefficients $\left\{\alpha_{i}\right\}$ are found from solving the Schrödinger equation $\mathscr{H}|\Psi\rangle=E|\Psi\rangle$, which reduces to a linear system of equations $\sum_{j} \mathscr{H}_{i j} \alpha_{j}=E \sum_{j} \mathcal{O}_{i j} \alpha_{j}$, for all $i$. Here, $\mathscr{H}_{i j}=\left\langle\phi_{i}|\mathscr{H}| \phi_{j}\right\rangle$ and $\mathcal{O}_{i j}=\left\langle\phi_{i} \mid \phi_{j}\right\rangle$ are the matrix elements of the Hamiltonian in the complete basis chosen and the overlapping matrix for the basis states, respectively.

In general, the number of basis states of the $N$-body problem increases exponentially with $N$, and the problem becomes untractable for values of $N$ which are rather small. To deal with large values of $N$, one is forced to truncate the complete basis set, and only retain a smaller subset of states which are most likely to contribute substantially in the decomposition of the ground-state wave function. The choice of this smaller subset is the crucial issue, since it determines the quality of the approximation. In what follows, we describe a particular subset which allows us to recapture certain key features of the exact Bethe ansatz solution of the 1D Hubbard model.

Consider the set of Slater determinants generated from the Hartree-Fock solution. Its states are $\left|\Psi_{\mathrm{HF}}\right\rangle$ and all possible particle-hole excitations $\left\{a_{\mathrm{h}}^{\dagger} a_{\mathrm{p}}\left|\Psi_{\mathrm{HF}}\right\rangle\right\},\left\{a_{\mathrm{h}}^{\dagger} a_{\mathrm{h}^{\prime}}^{\dagger} a_{\mathrm{p}} a_{\mathrm{p}^{\prime}}\left|\Psi_{\mathrm{HF}}\right\rangle\right\}$, etc. Obviously, if all possible combinations of occupied and empty orbitals are considered, the set thus spanned is a complete basis of the $N$-body space.

Let us now order the states in this HF basis set according to their energies $\langle\phi|\mathscr{H}| \phi\rangle$. If we are interested in the ground state and the low-lying excitations of the system, we only need to keep the low-energy states of the HF basis set. This procedure is, in fact, very well known for Hamiltonians with a non-degenerate $\mathrm{HF}$ ground state. If only the HF ground state and the states with one particlehole excitations are kept, this leads to the random phase approximation (RPA). Besides a better approximation for the ground state than simple HFA, the RPA enables us to find collective excitations and the particle-hole continuum.

On the other hand, the doped HF ground states of the spin-flux Hamiltonian are degenerate. For instance, the meron-antimeron pair shown in Fig. 5 happens to be centered at site $(10,10)$. It is obvious that configurations which have the meron-antimeron pair centered about any other site will have 
the exact same HF energy (cyclic boundary conditions are imposed). Also, if the center for the pair is fixed at a site, there are four distinct possible arrangements of the meron and antimeron about that site, obtained by rotating the meron-antimeron axis by $90^{\circ}$. Thus, for a plaquette of size $N_{x} \times N_{y}$, the HF ground state is $4 N_{x} N_{y}$ degenerate. Clearly, the minimal choice for the subset of states used to search for the ground state of the doped system must include all these degenerate HF ground-state wave functions. This choice is the essence of the configuration interaction (CI) approximation [25]. One expects these HF states to mix with equal weight $\left|\alpha_{i}\right|^{2}$ in the decomposition of the ground state and low-energy states $|\Psi\rangle=\sum_{i=1}^{4 N_{x} N_{y}} \alpha_{i}\left|\phi_{i}\right\rangle$. Specifically, we denote $\left|\phi_{i}\right\rangle \rightarrow\left|\Psi_{\theta}(n, m)\right\rangle$ to be the HF ground-state wave function describing a configuration centered at site $(n, m)$ of the lattice, and with the meron-antimeron axis at an angle $\theta$ from the $x$-axis. Then, $1 \leqslant n \leqslant N_{x}, 1 \leqslant m \leqslant N_{y}$ and $\theta=45^{\circ}, 135^{\circ}, 225^{\circ}$ or $315^{\circ}$. On general symmetry grounds one expects the ground state and the lowlying energy states to have the general form

$\left|\Psi_{J}(\boldsymbol{k})\right\rangle=\sum_{n, m, \theta} \mathrm{e}^{\mathrm{i}\left(k_{x} n+k_{y} m\right) a} \mathrm{e}^{\mathrm{i} J \theta}\left|\Psi_{\theta}(n, m)\right\rangle$.

The cyclic boundary conditions limit the vector $\boldsymbol{k}$ to a subset of equally spaced values inside the first Brillouin zone and $J$ must be an integer.

Eq. (4) shows that the ground state and low-lying energy states found within the CI method have translational and rotational symmetry. This procedure overcomes the most glaring shortcoming of the mean-field theory (the broken translational and rotational invariance). Clearly, the CI wave functions describe quantum dynamics of the charge carriers. The pair is no longer pinned at one site, as in HFA, but moves over the entire lattice. The mobility of the pair and its preferred internal angular momentum can be obtained from the dispersion relation $E_{J}(\boldsymbol{k})=\left\langle\Psi_{J}(\boldsymbol{k})|\mathscr{H}| \Psi_{J}(\boldsymbol{k})\right\rangle$. The lowest energy band of the meron-antimeron pair obtained for $U / t=5$ is shown in Fig. 6 [23]. We find that the CI ground state corresponds to pairs of total momentum $(\pi / a, \pi / a)$, and which have d-wave symmetry, $J=2$. In fact, $J$ varies throughout the Brillouin zone as shown in Fig. 7, from pure d-wave around the $( \pm \pi / a, \pm \pi / a)$ points to pure s-wave

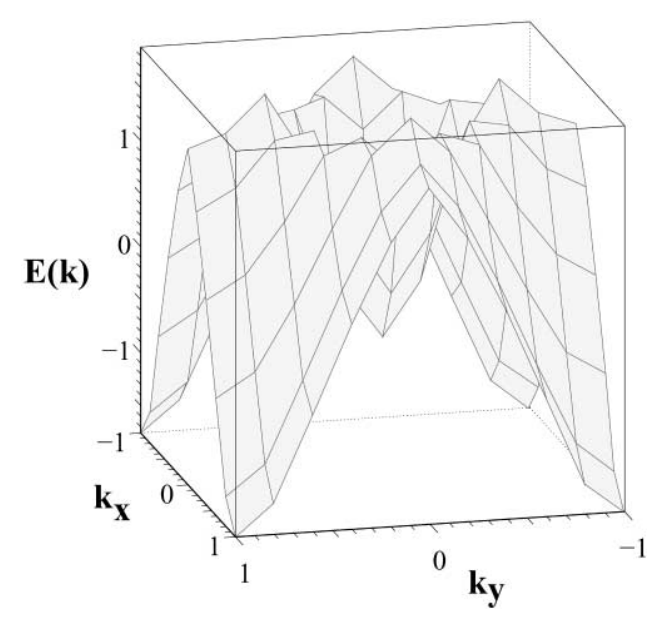

Fig. 6. The lowest energy dispersion band $E_{J}(\boldsymbol{k})$ (in units of $t$ ) as a function of the total momentum $\boldsymbol{k}$ of the meron-antimeron pair. The momentum units are $\pi / a$ and $U / t=5$. For convenience, the reference energy is taken to be the static HF energy of the self-consistent meron-antimeron pair. Quantum hopping and rotation lowers the overall energy of the pair by $1.75 t$.

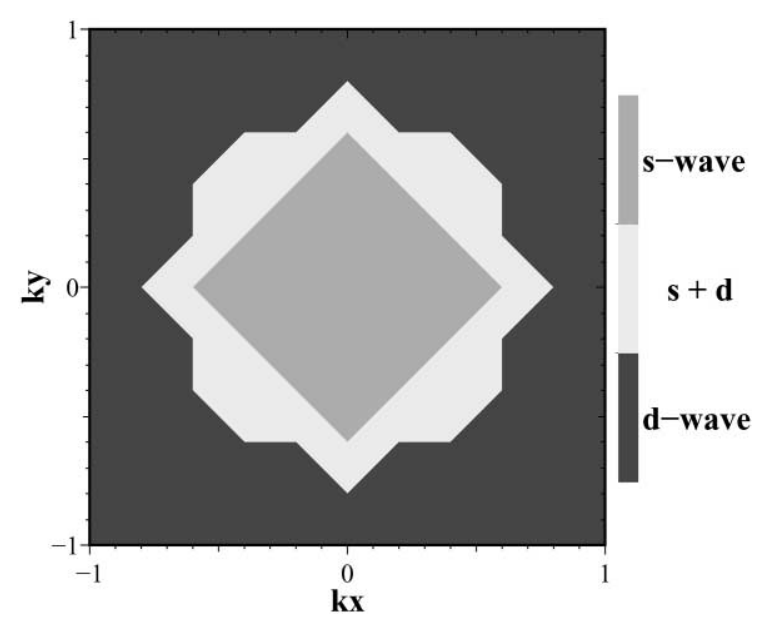

Fig. 7. The rotational symmetry of the meron-antimeron wave function as a function of the total momentum carried by the pair (measured in units of $\pi / a$ ). The outside region (containing the absolute minima points $(\pi, \pi))$ has d-wave symmetry $(J=2)$, while the core region about the $(0,0)$ point has s-wave symmetry $(J=0)$. The intermediary area is a mix of $s+d$ wave symmetry.

around the $(0,0)$ point, where a second local minimum exists. The large width $(\approx 4 t)$ of the dispersion band clearly proves that the meron-antimeron pair is a very mobile excitation. The existence of 


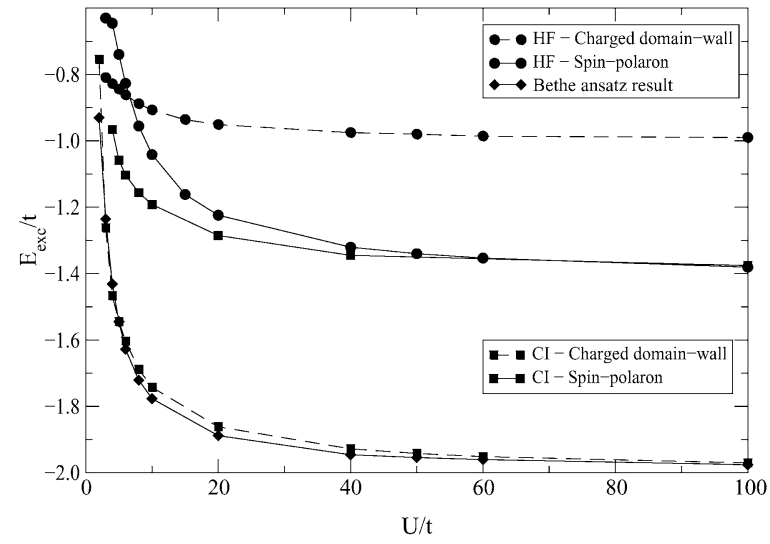

Fig. 8. Excitation energy, in units of $t$, for a mobile charged domain wall (squares, dashed line) and a mobile charged spinpolaron (squares, full line), as obtained from the CI approach. The exact excitation energy given by the Bethe-ansatz method is shown by diamonds. The domain-wall CI energy is in excellent agreement with the exact $\mathrm{BA}$ results overt the entire $U / t$ range, while the spin-polaron CI energy is significantly different. For comparison, we also show the excitation energies for the domain wall (circles, dashed line) and the spin-polaron (circles, full line) as obtained within HFA, proving again that the extra kinetic energy gained by the moving domain wall is of order $t$ for most $U / t$ values. In contrast, the extra kinetic energy gained by the spin-polaron is of order $t^{2} / U \rightarrow 0$ as $U / t$ increases, so in the large $U / t$ limit there is almost no difference between the HF and CI results for the charged spin-polaron. We conclude that the charged domain-wall is the relevant excitation for all values of $U / t$.

mobile charged bosonic merons and antimerons thus provides a microscopic basis for the nonFermi liquid "parent" metal from which superconductivity emerges.

As an indication of the validity and accuracy of the CI method, we briefly review the results of such an analysis of the 1D Hubbard model [12]. Here, the analog of the a charged meron-vortex is the charged domain wall soliton which facilitates a $\pi$ flip from one AFM ground state to the other AFM ground state (with all spins flipped). The charged domain wall traps the hole on a pair of levels that are localized deep inside the MottHubbard gap, and is a charged boson [12,21,22] The analog of the spin-bag is the spin-polaron, which disturbs the AFM order only locally, trapping the hole in its small FM core. As a result, the spin-polaron carries both charge and spin- $\frac{1}{2}$. In
Fig. 8, we show the HF (circles) and CI (squares) energies of both the spin-polaron (full line) and the charged domain-wall (dashed line). For the spinpolaron, the difference between the $\mathrm{HF}$ and $\mathrm{CI}$ energy varies as $t^{2} / U$. In analogy with the $2 \mathrm{D}$ case, this is the expected behavior since the spin $-\frac{1}{2}$ carried by the spin-polaron localizes it on one magnetic sublattice. On the other hand, the domain wall lowers its energy by an amount of the order $t$ between the HF and the CI values. This clearly shows that the charged domain wall is a very mobile objects, and that its dynamics must be properly described in order to get a realistic picture. When this is done, it is apparent that the charged domain wall is the low-energy excitation over the entire $U / t$ range, and in fact its energy is in excellent agreement with the exact Bethe Ansatz prediction (shown as diamonds).

Finally, we consider the stability of the ground state and the low-lying energy states to inclusion of more states in the subset used to generate them. The next meaningful enlargement of this subset is to add to it all states with one particle-hole excitation, obtained from all degenerate HF ground-state wave functions. While this increases the size of the subset substantially, it does not lead to a lower ground-state energy. This is because the system is gapped. The lowest energy particle-hole excitation is obtained when an electron from the top of the valence band is excited on one of the bound levels, localized in the cores of the meron and the antimeron. These levels are roughly one quarter of the way into the gap (for $U / t=5$ ), so the states with a particle-hole excitation have an energy of order US more than the HF ground states. Consequently they do not mix into the ground state. They only influence the higher-energy states. The low-lying excitations depicted in Fig. 6 are charge excitations, associated with motion of the charged meron-antimeron pair. These are not affected by addition of excited states in the variational subset, except at rather high energy. But there also exist low-lying spin-wave excitations, which are described by adding particle-hole excited states (RPA) in the variational subset. While it is very likely that the nucleation of merons and antimerons in the AFM background will alter the dispersion of the spinwaves, it is interesting to note that low-lying charge 
and low-lying spin excitations have very different origin. This is related to the spin-charge separation tendency exhibited by this system.

If a single hole is added to the 2D AFM plane and the cyclic boundary conditions are imposed, the HF approximation leads to a spin-bag meanfield solution for the spin-flux phase. We analyze the translational properties of the spin-bag using the CI method. As it turns out [23], the spin-bag is a very immobile object. The width of its dispersion band is of the order of $t^{2} / U$, i.e. much smaller than the $\sim t$ bandwidth of the meron dispersion. The reason is that the spin-bag carries both charge and spin $-\frac{1}{2}$. The spin- $-\frac{1}{2}$ only allows the spin-bag to live on one of the magnetic sublattices. (If it were to move to the other sublattice, its spin should flip, and such processes are forbidden). In order to move, a spin-bag must tunnel two sites to a second-nearest-neighbor site, and this is a $t^{2} / U$ process. On the other hand, the charged meron carries no spin, so there are no restrictions for its motion. It can move to a nearest-neighbor site, leading to a $t$-hopping process. This suggests that when dynamics is properly taken into consideration within CI, a highly mobile vortex-antivortex pair sharing a single hole is energetically favored to the immobile spin-bag. We have verified this hypothesis, and demonstrated that an upper bound to the energy of a singly charged vortex-antivortex pair is, indeed, much lower than the CI energy of spin-bag [23]. The charged spin-bag solution is thus unstable to dissociation into a highly mobile vortex-antivortex pair, which shares the hole. When a second hole is added, a second single-charged vortex-antivortex pair is nucleated. However, it becomes energetically more convenient for the two holes to become bound to the same vortex-antivortex pair, leading to the appearance of a meron-antimeron pair which carries both charges (pre-formed Dwave pair). The remaining uncharged vortexantivortex pair is unstable to collapse, at low temperatures.

\section{Discussion and comparison with experiments}

The crucial distinguishing feature of our model is the concept of spin-flux, the dynamical possibility of an electron undergoing a somersault as it traverses a closed loop. In the undoped parent compound, this leads to dispersion relations with isotropic symmetry about the Fermi point, in excellent agreement with those measured experimentally through ARPES. This symmetry of the dispersion relations leads to real-space configurations which have the same type of symmetry. In the spin-flux model, the holes doped into the AFM plane nucleate magnetic vortices and become trapped in their cores, leading to the appearance of mobile, bosonic, charge carriers.

In contrast, both the conventional Hubbard model and its asymptotic limit, the $t-J$ model, exhibit a very large, nested Fermi surface (at the mean-field level) in the undoped parent compound. This Fermi surface has quasilinear 1D character, since there is no $k$-dependence along the Fermi surface. As a result, configurations stabilized by doping exhibit the same quasi-1D character in real space. It has been suggested they take the form of charged stripes [26-31]. We note finally that vortex-like configurations are unstable in the conventional Hubbard model, while stripe-like configurations are generally unstable to the formation of a quantum liquid of merons in the spin-flux model. One notable exception is the commensurate case $x=0.125$, when merons and antimerons crystallize along 1D lines, leading to the stripe-like configuration observed experimentally [32], provided that a small $(3 \%)$ hopping anisotropy is included in the model [17].

The mobile bosonic charged meron-vortices created through doping provide a microscopic basis for a non-Fermi-liquid behavior. They also exhibit a very strong pairing attraction, of magnetic origin. This pairing, which arises in a purely repulsive electron system, leads to appearance of pre-formed "Cooper-pairs" of d-wave symmetry. This agrees with the experimental findings of d-wave superconductivity and the scaling of the superfluid density with doping. The pre-formed pairs may also be related to the observation of a pseudo-gap on the underdoped side of the phase diagram [33-35].

Many other features of our model are in agreement with experimentally observed properties of the cuprate superconductors. Nucleation of magnetic vortices with doping explains a variety of 
magnetic properties, starting with complete destruction of the long-range AFM order for very low doping concentration. As we can see from Fig. 5, a tightly bound meron-antimeron pairs disturbs the long-range AFM ordering of about 100 spins of the lattice. For very low dopings, these pairs are far from each other, and there are many spins on the plane whose orientations are not affected by any pair. Thus, most of the spins maintain the longrange AFM order. However, as the doping increases over about $2 \%$, the areas occupied by each meron-antimeron pairs start to overlap with those occupied by the neighboring pairs. At this doping the orientation of all the spins on the $\mathrm{CuO}_{2}$ planes is affected by at least one pair of vortices, and therefore the LRO is lost. The local ordering, however, is still AFM. This picture explains the extremely low doping necessary for the disappearance of LR AFM order, as well as the fact that the spin correlation length is basically equal to the average distance between holes (vortices) and does not depend strongly on the temperature [10]. Each hole carries its vortex with it, and the spins in each vortex are correlated with each other. The correlation length is thus roughly equal to the average inter-vortex (inter-hole) distance. The nucleation of magnetic vortices quantitatively explains the split of the $(\pi, \pi)$ AFM Bragg peak into the four incommensurate peaks whose positions shift with doping [11], as observed in $\mathrm{LaCuO}$ and, more recently, in $\mathrm{YBaCuO}$ [36]. The form factor of any given vortex already gives rise to an apparent splitting of the neutron scattering peak. As demonstrated in Ref. [14], even at the mean-field level we recapture the neutron scattering data using the HF distribution of meron-vortices. A more detailed investigation suggests that saturation [37] of the peak splitting for $x>0.12$ may be related to expansion of the core radius of the individual vortices at higher doping [38].

Optical behavior of the cuprates is also explained naturally using our model. Two features develop in the optical absorption spectra with doping: a broad mid-infrared temperature-independent absorption band, and a strongly temperature-dependent lowfrequency Drude tail [8]. In our model the broad mid-infrared band is related to excitation of electrons from the valence band onto the empty levels bound in the vortex cores [17], which are localized approximately one quarter of the way inside the Mott-Hubbard gap. The number of localized levels scales with the number of vortices, and inter-vortex and spin-wave interactions lead to their broadening into the observed band. This mechanism is similar to the one leading to a broad mid-infrared absorption band in polyacetylene with doping [39]. (In the continuum limit, the meron-vortex in fact creates a pair of mid-gap states in the Mott-Hubbard gap [21]). The polyacetylene band is due to electronic excitations inside the cores of the polyacetylene domain-wall solitons [24], which are the topological analogues of meron-vortices [21,22]. Another strong argument in favor of this interpretation is provided by photoinduced absorption experiments [40]. If the undoped parent compounds are illuminated with intense visible light, they develop absorption bands that resemble the mid-infrared bands of the doped compounds. Similar behavior is observed in polyacetylene, and is attributed to the nucleation of solitons by photoexcited electron-hole pairs [41].

The second component of the optical spectrum is the Drude tail. It results from the response of the freely moving charged vortices to the external electric field. The strong temperature dependence of this tail is determined by the scattering mechanism for merons (due to interactions with other merons and spin-waves). This interpretation is also supported by the fact that the superconducting transition leaves the mid-infrared absorption band unchanged. Merons with internal electronic structure are still present on the planes but pair condensation leads to a collapse of the Drude tail into a $\delta(\omega)$ response.

Finally, our model provides some understanding of the cross-over towards the Fermi-liquid metal in the overdoped phase. For large dopings $(\delta>0.30-0.40)$ the average inter-vortex spacing becomes extremely small and the very cores of the merons start to overlap. In this limit the MottHubbard gap is completely filled in by the discrete levels, and the spin-flux state becomes energetically unstable relative to a normal Fermi liquid [17].

We note, finally, that the spin-flux Hamiltonian has essentially no free or adjustable parameters. The choice of $U / t$ is fixed by the experimentally 
measured size of the Mott-Hubbard charge transfer gap at zero doping. All other experimental features such as (i) the position and nature of the mid-infrared optical absorption band, (ii) the ARPES data and (iii) the position of the magnetic neutron scattering satellite peaks as a function of doping, are quantitatively described by the same choice. More detailed comparisons with specific experiments may require the incorporation of specific (smaller energy scale) interactions which are not included in this simplest version of the spinflux Hamiltonian.

\section{Acknowledgements}

M.B. acknowledges support from a Natural Sciences and Engineering Research Council of $\mathrm{Ca}-$ nada Postdoctoral Fellowship.

\section{References}

[1] J.G. Bednorz, K.A. Muller, Z. Phys. B 64 (1986) 189.

[2] P.W. Anderson, Phys. Rev. 115 (1959) 2.

[3] Y. Iye, in: D.M. Ginsberg (Ed.), Physical Properties of High Temperature Superconductors III, World Scientific Press, Singapore, 1992.

[4] S. Martin et al., Phys. Rev. B 41 (1990) 486.

[5] S.J. Hagen et al., Phys. Rev. B 37 (1988) 7928.

[6] T.A. Friedmann et al., Phys. Rev. B 42 (1990) 6217.

[7] N.W. Aschroft, N.D. Mermin, Solid State Physics, Saunders College Publishing, London, 1976.

[8] D.B. Tanner, T. Timusk, in: D.M. Ginsberg (Ed.), Physical Properties of High Temperature Superconductors III, World Scientific Press, Singapore, 1992.

[9] N.P. Ong, in: D.M. Ginsberg (Ed.), Physical Properties of High Temperature Superconductors II, World Scientific Press, Singapore, 1990.

[10] R.J. Birgeneau, G. Shirane, in: D.M. Ginsberg (Ed.), Physical Properties of High Temperature Superconductors I, World Scientific Press, Singapore, 1989.
[11] K. Yamada et al., Phys. Rev. B 57 (1998) 6165 and references therein.

[12] M. Berciu, S. John, Phys. Rev. B 61 (2000) 10015.

[13] N. Rosen, Phys. Rev. 82 (1951) 621.

[14] S. John, A. Golubentsev, Phys. Rev. Lett. 71 (1993) 3343.

[15] S. John, A. Golubentsev, Phys. Rev. B 51 (1995) 381.

[16] S. John, A. Müller-Groeling, Phys. Rev. B 51 (1995) 12989.

[17] M. Berciu, S. John, Phys. Rev. B 59 (1999) 15143.

[18] B.O. Wells et al., Phys. Rev. Lett. 74 (1995) 964.

[19] S. LaRosa et al., Phys. Rev. B 56 (1997) R525.

[20] C. Kim et al., Phys. Rev. Lett. 80 (1998) 4245.

[21] S. John, M. Berciu, A. Golubentsev, Europhys. Lett. 41 (1998) 31.

[22] M. Berciu, S. John, Phys. Rev. B 57 (1998) 9521.

[23] M. Berciu, S. John, Phys. Rev. B 61 (2000) 16454.

[24] A.J. Heeger, S. Kivelson, J.R. Schrieffer, W.-P. Su, Rev. Mod. Phys. 60 (1988) 781.

[25] E. Louis et al., Phys. Rev. B 59 (1999) 14005.

[26] D. Poilblanc, T.M. Rice, Phys. Rev. B 39 (1989) 9749.

[27] H.J. Schultz, J. Phys. (Paris) 50 (1989) 2833.

[28] M. Kato, K. Machida, H. Nakanishi, M. Fujita, J. Phys. Soc. Jpn. 59 (1990) 1047.

[29] K. Machida, Physica C 158 (1989) 192.

[30] J. Zaanen, O. Gunnarson, Phys. Rev. B 40 (1989) 7391.

[31] J. Zaanen, M.L. Horbach, W. van Saarloos, Phys. Rev. B 53 (1996) 8671.

[32] J.M. Tranquada et al., Nature (London) 375 (1995) 561.

[33] T. Timusk, B. Statt, Rep. Prog. Phys. 62 (1999) 61.

[34] V.J. Emery, S.A. Kivelson, Nature 374 (1995) 4347.

[35] V.J. Emery et al., Phys. Rev. B 56 (1997) 6120.

[36] M. Arai et al., Phys. Rev. Lett. 83 (1999) 606.

[37] G. Xu et al., Science 289 (2000) 419.

[38] M. Berciu, S. John, in preparation.

[39] J. Tanaka, M. Tanaka, Optical spectra of conducting polymers, in: T.A. Skotheim (Ed.), Handbook of Conducting Polymers II, Marcel Dekker Inc., New York, 1986.

[40] C.M. Foster, A.J. Heeger, G. Stuckey, N. Heron, Solid State Commun. 71 (1989) 945.

[41] J. Orenstein, Photoexcitation of conjugated polymers, in: T.A. Skotheim (Ed.), Handbook of Conducting Polymers II, Marcel Dekker Inc., New York, 1986. 